\title{
ENERGY EFFICIENT MULTICAST ROUTING IN MANET
}

\author{
Sankar $\mathrm{M} \mathrm{S}^{1}$ and Suganthi B ${ }^{2}$ \\ $1 \& 2$ Department of Electronics and Communication, Dhanalakshmi Srinivasan \\ Engineering College, Perambalur, Tamil Nadu, India. \\ sankaraswin@gmail. $\mathrm{com}^{1}$ \\ suguadi@sify. $\mathrm{com}^{2}$
}

\begin{abstract}
In this paper, we have presented the Modified Multicasting through Time Reservation using Adaptive Control for Excellent Energy efficiency (MMC-TRACE). It is a real time multicasting architecture for Mobile Ad-Hoc networks to make their work an energy efficient one.MMC-TRACE is a cross layer design where the network layer and medium access control layer functionality are done in a single integrated layer design. The basic design of the architecture is to establish and maintain an active multicast tree surrounded by a passive mesh within a mobile ad hoc network. Energy efficiency is maximized by enabling the particular node from sleep to awake mode while the remaining nodes of the same path are maintained at sleep mode. Energy efficiency too achieved by eliminating most of the redundant data receptions across nodes. The performance of MMC-TRACE are evaluated with the help of ns-2 simulations and comparisons are made with its predecessor such as MC-TRACE. The results show that the MMC-TRACE provides superior energy efficiency, competitive QoS performance and bandwidth efficiency.
\end{abstract}

\section{KEYWORDS}

Quality of Service, Cross Layer Design, Multicast Routing, Redundant Data Detection.

\section{INTRODUCTION}

QoS is the performance level of a service offered by the network, in general. Specifically, QoS in voice communications necessitates 1) maintaining a high enough packet delivery ratio (PDR), 2) keeping the packet delay low enough, and 3) minimizing the jitter in packet arrival times. Thus, the goal in QoS provisioning is to achieve a more deterministic network behaviour (i.e., bounded delay, jitter, and PDR) .Actually, flooding, which is the simplest group communication algorithm, is good enough to achieve high PDR provided that the data traffic and/or node density is not very high so that the network is not congested. However, flooding generally is not preferred as a multicast routing protocol due to its excessive use of the available bandwidth. Thus, the second objective of a multicast routing protocol is to utilize the bandwidth efficiently, which is directly related with the number of retransmissions (throughout this paper, the term retransmission is used for relaying) required to deliver generated data packets to all members of a multicast group with a high enough PDR. The third objective of a multicast protocol is to minimize the energy dissipation of the network. Although optimizing the performance of a wireless communication system by incorporating cross-layer design is a tempting choice, several researchers have argued that such a cross-layer design is not the best choice in the long run because it sacrifices modularity and can lead to unintended cross-layer interactions. However, by strictly adhering to a

DOI : 10.5121/ijcseit.2012.2304 
standard layering approach, we may miss out on performance improvements that can be offered through the exploitation of the less restricted cross-layer design space. Therefore, in this paper, we propose a multicasting architecture based on cross-layer design while exercising the utmost caution to avoid unintended cross layer interactions. MC-TRACE inherits its cross-layer architecture from the MH-TRACE architecture. Although there are many protocols for multicasting in mobile ad hoc networks], to the best of our knowledge, there is no single protocol that jointly addresses QoS, spatial reuse efficiency, and total energy dissipation.

\section{RELATED WORK}

There are many multicast routing protocols designed for mobile ad hoc networks they can be categorized into two broad categories: tree-based approaches and mesh-based approaches. Treebased approaches create trees originating at the source and terminating at multicast group members with an objective of minimizing a cost function. A multicast protocol for ad hoc wireless networks (AMRIS) constructs a shared delivery tree rooted at one of the nodes, with IDs increasing as they radiate from the source. Local route recovery is made possible due to this property of the IDs, hence, reducing the route discovery time and also confining route recovery overhead to the proximity of the link failure. Mesh-based multicasting is better suited to highly dynamic topologies, simply due to the redundancy associated with this approach.. In mesh-based approaches, there is more than one path between the source and the multicast group members (i.e., a redundant multicast tree). One such mesh-based multicast protocol, On-Demand Multicast Routing Protocol (ODMRP), is based on periodic flooding of the network by the source node through control packets to create a multicast mesh. This basic operation is used both to create the initial multicast forwarding state and to maintain the mesh in case of node mobility and other network dynamics. In ODMRP, an active source periodically floods the network with JOIN QUERY control packets. When a node receives a JOIN QUERY packet, it marks the first node it receives the packet from as the upstream node and rebroadcasts the JOIN QUERY packet. When a multicast group member receives a JOIN QUERY packet, it replies back with a JOIN REPLY packet, which is forwarded back to the source node via traversing the reverse path. Each upstream node sets a group forwarding flag for the multicast group indicated in the packet header and becomes a member of the multicast mesh. The forwarding state expires after a predetermined time. The MC-TRACE active multicast backbone is a highly pruned tree. In, an energy efficient multicasting algorithm for wireless networks with fixed transmit power nodes is proposed. In , a passive clustering algorithm, which considers both stability and residual energy of neighbouring nodes when selecting cluster heads and gateways, is proposed. This algorithm significantly reduces routing-related control overhead. The focus of the model is on multicast services in wireless LANs.

\subsection{MC-TRACE MEDIUM ACCESS CONTROL ARCHITECTURE}

Multicasting through Time Reservation using Adaptive Control for Energy efficiency (MCTRACE) is a MAC protocol for energy-efficient real-time data communications In MC-TRACE, the network is partitioned into overlapping clusters through a distributed algorithm. Time is organized into cyclic constant duration super frames ð TSF P consisting of several frames. Each cluster head $(\mathrm{CH})$ chooses the least noisy frame to operate within and dynamically changes its frame according to the interference level of the dynamic network. Nodes gain channel access through a dynamically updated and monitored transmission schedule created by the $\mathrm{CHs}$, eliminating packet collisions within the cluster. Collisions with the members of other clusters are also reduced by the CH's selection of the minimal interference frame. Nodes that are scheduled to transmit data send a short IS packet prior to data transmission. The IS packet includes information about the data packet, for example, in an IS slot, the ID of the corresponding upcoming data packet 1 is announced so that the nodes that have already received the data packet do not waste 
energy receiving a previously received data packet. Channel access is automatically renewed by the continuous use of a reserved data slot.

\subsection{MMC-TRACE ARCHITECTURE}

The basic functionalities provided by MC-TRACE are topology control, cluster creation and maintenance, distributed data transmission scheduling, and medium access control. MC-TRACE have the mechanism for routing but lacks error handling and maintenance. MMC-TRACE is an architecture built on MC-TRACE and is capable of multicast routing and error handling. Both the advantages provided by and the restrictions imposed by MC-TRACE on MMC-TRACE are explained

\subsection{Integration with MH-TRACE}

Layer part, where the network layer functionality is tailored according to the MAC layer functionality. The integration principles of MMC-TRACE with MC-TRACE can be itemized as follows:

1. MC-TRACE is designed to provide periodic channel access to a stream of data packets (to provide QoS for streaming media, like real-time voice or video sources). Thus, once channel access is granted to a node, the channel should be utilized for a group of periodic data packets rather than a single data packet; otherwise, the efficiency of the channel access is deteriorated. Furthermore, data slots are designed to host relatively large chunks of data in MMC-TRACE to provide better scheduling, and consequently, better energy efficiency for mobile nodes. If a data slot is used to transmit a control packet, then most of the slot time will be empty and bandwidth will be wasted. The projection of these facts on the design of MMC-TRACE is that the main multicast tree creation and maintenance mechanisms should be carried out through IS packets rather than through independent control packets transmitted in data slots. Hence, it is more efficient to reserve the data slots for data packets and use other mechanisms to transmit control packets.

2. Header packets include the data transmission schedule of the coming frame, which is a common feature of many TDMA-based MAC protocols, including the IEEE 802.16. This information can be used by the nodes that receive the header packet to schedule the reception times of the data packets they are interested in. However, in a multihop network, not all nodes will hear the header packet sent by their neighbours' $\mathrm{CH}$, and thus, they will not know when their neighbours are scheduled to transmit. Relaying the schedule packet to two-hop neighbours could be a solution, but this will incur extra overhead. One solution is to have every node that is scheduled to transmit data in the current frame transmit its own schedule prior to data transmission. MH-TRACE provides a framework that can be utilized for this purpose, through the use of the IS slots. The IS packet format is presented.

3. In some cases, network operation necessitates explicit control functionality beyond what can be performed implicitly through data packets. For such cases, control packets are needed. The IS sub frame is the most appropriate portion of the frame in which to transmit such packets, as all the nodes are guaranteed to be awake only during the IS sub frame. The aforementioned integration principles outline both the design limitations imposed and the benefits provided by the underlying MC-TRACE MAC scheme in MMC-TRACE.

\subsection{Overview}

There are five basic building blocks in MMC-TRACE as follows: 
1. Route discovery (RTD)

2. Route allocation (RTA)

3. Route maintenance (RTM)

4. Route error handling, (REH) and

5. Route creation (RTC)

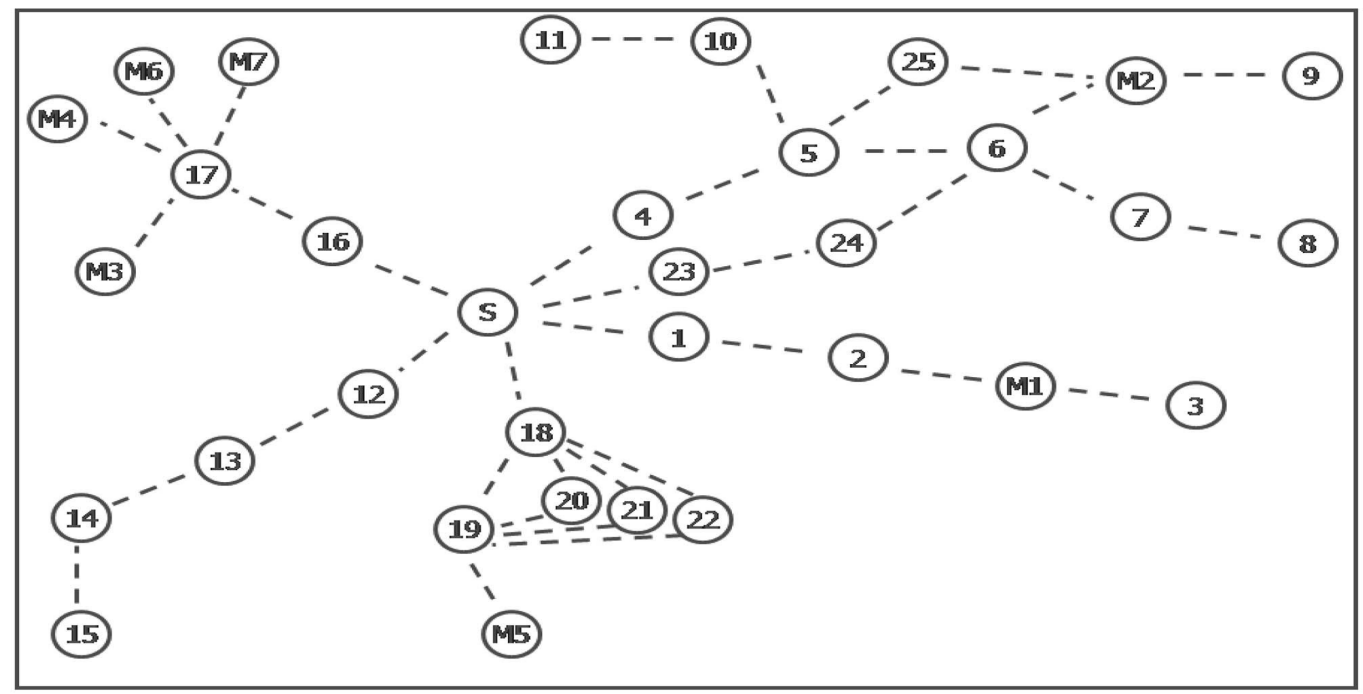

Figure 1: Multicast Group Node Allocation Representation

\subsubsection{ROUTE DICOVERY:}

A source node initiates a session by broadcasting packets to its one-hop neighbours. Nodes that receive a data packet contend for channel access, and the ones that obtain channel access retransmit the data they received. Eventually, the data packets are received by all the nodes in the network, possibly multiple times. Each retransmitting node acknowledges its upstream node by announcing the ID of its upstream node in its IS packet, which precedes its data packet transmission. Such a scenario can be better understood by considering the network branch formed by nodes S, 1, 2, M1, and 3 in Fig. The contents of the IS packets for this network branch are illustrated in Fig. 2a. The source node announces its own ID as its upstream node ID. Initially, all retransmitting nodes announce the null ID as their downstream node ID. However, when an upstream node is acknowledged by a downstream node, the node updates its downstream node ID by the ID of this node. The leaf nodes (i.e., nodes that do not have any downstream nodes that are acknowledging them as upstream nodes) continue to announce the null ID as their downstream node ID. At this point, some of the nodes have multiple upstream nodes (i.e., multiple nodes that have lower hop distance to the source than the current node) and downstream nodes (i.e., multiple downstream nodes acknowledging the same upstream node as their upstream node). A node updates its own HDTS by incrementing the least HDTS it hears within THDTS1 time. The initial HDTS value is set to HDTSMAX, and the HDTS value is again set to HDTSMAX if a node does not receive any IS or data packets for more than THDTS2 time (THDTS2 > THDTS1). Multicast group member nodes indicate their status by announcing their multicast group ID in the IS packet.

\subsubsection{ROUTE ALLOCATION}

A node that is not a multicast relay also ceases to retransmit data if it does not receive an ACK3 from any downstream node. After the initial flooding, all the nodes receive the data packets and 
they determine their upstream and downstream nodes. Multicast relays are also determined. Nodes 1, 2, and M1 along with S are multicast relays. However, nodes 12, 13, 14, and 15 are not multicast relays because there is no multicast group member connected to that branch of the network (as described in the previous section, node 3 is also not a multicast relay). Node 15 will cease retransmitting the packets that it receives from its upstream node 14 TRLY time after its first retransmission of data because no node is acknowledging its data transmissions. However, until that time, node 15 acknowledges its upstream node, which is node 14. Some of the multicast group members are not multicast relays. The left panel of Fig. 2c illustrates such a situation. Multicast node (node M3) is a multicast relay, as indicated by the two-way arrows; whereas nodes M4, M6, and M7 are not multicast relays-they just receive packets from the upstream node (node 17). Hence, nodes M4, M6, and M7 do not acknowledge node 17 (node 17 is acknowledged by node M3). Note that any node can acknowledge only one upstream and one downstream node with a single IS packet. When node M3 moves away from node 17's transmit range and enters node 16's transmit range, it either begins to acknowledge node 16 as its upstream node if the transition happens in less than TRLY time (i.e., node M3's multicast relay status does not expire before TRLY time), or node M3 just receives the data packets from node 16 without acknowledging node 16 if node M3's transition takes more than TRLY time. In any case, node 17 does not receive any ACK from node M3 for TACK time and starts to set its downstream node ID as the null ID. However, node 17 does not cease retransmitting data packets that it receives from its upstream node (node 16) instantly because a multicast relay does not reset its status for TRLY ð TRLY > TACKP time, and thus, continues to retransmit data packets. Although none of the other multicast nodes acknowledge any node, they monitor their upstream node through IS and data packets.

\subsubsection{ROUTE MAINTAINENCE:}

When the upstream node of one or multiple multicast group member node(s) announces the null ID as its downstream node ID, the multicast nodes (nodes M4, M6, and M7) start to acknowledge the upstream node by announcing the ID of the upstream node (node 17) in their IS packets. Thus, node 17 continues to be a multicast relay and one of the downstream multicast nodes (node M4 in this scenario) becomes a multicast relay after receiving a downstream ACK from its upstream node (node 17) acknowledges node M4 by a downstream ACK because it is the first node that acknowledges node 17 by an upstream ACK - the other multicast nodes' ACKs are received by node 17 later than the ACK of node M4). Observe that nodes M4, M6, and M7 form a redundant passive outer mesh for the tree branch. The RTM mechanism does not necessarily create a new branch, yet it prevents an existing branch from collapsing. However, there are situations where new branches should be incorporated into the tree.

\subsubsection{ROUTE ERROR HANDLING:}

After a node marks itself as a multicast relay, it continuously monitors its upstream node to detect a possible link break between itself and its upstream multicast relay node, which manifests itself as an interruption of the data flow without any prior notification. If such a link break is detected, the downstream node uses the RPB mechanism to fix the broken link. Fig.. illustrates an example of a network topology, where a branch of the multicast tree is broken due to the mobility of a multicast relay and then fixed by the REH mechanism. The left panel of Fig. $4 \mathrm{~d}$ shows a multicast tree formed by the source node, node $S$, multicast relay nodes, nodes 18 and 19 , and the multicast group node, node M5, which is a multicast relay as well. Nodes 20, 21, and 22 are neither multicast relay nodes nor multicast group members; however, they receive the IS packets from nodes 18, 19, and M5 (i.e., nodes 20, 21, and 22 are in the receive ranges of all the three nodes). After some time, as illustrated in the right panel of Fig. 4d, node 19 moves away from its original 
position and nodes 18 and 19 cannot hear each other; thus, the multicast tree is broken. However, nodes 19, 20, 21, 22, and M5 can hear each other, temporarily.

\subsubsection{ROUTE CREATION:}

It is possible that due to the dynamics of the network (e.g., mobility, unequal interference), a complete branch of a multicast tree can become inactive, and the leaf multicast group member node cannot receive data packets from the source node. Fig. illustrates a network with one active branch, composed of nodes S, 1, 2, and M1, and one inactive branch, composed of nodes 4, 5, 6, and M2 (assume that nodes 23, 24, and 25 are passive nodes and they are pruned down after IFL). The double arrows indicate an active link with upstream and downstream ACKs. Dashed lines indicate an inactive link. The numbers below the nodes show their HDTS, which are acquired during previous data transmissions. One situation that can create such inactivity is that the upstream ACKs of node 77 and node M2 are colliding and node 6 cannot receive any downstream ACK. Thus, node 6 ceases to relay packets, which eventually results in silencing all the upstream nodes up to the source (i.e., if node 6 does not get any downstream ACKs, it ceases acknowledging its upstream node, node 5, after TRLY time, which results in the silencing of node 5 in 2TRLY time and node 4 in 3TRLY time). If a multicast group member, node M2 in this scenario, detects an interruption in the data flow for TCRB time, it switches to Create Branch status and announces this information via a RTM packet. Note that such a node first tries repairing the branch through the RTM mechanism, and it will use the RTM mechanism only if the RTM mechanism fails to fix the interruption of data flow for TCRB time. A RTM packet is transmitted by using one of the empty IS slots, which is chosen randomly. Upon receiving a RTM packet, all nodes in the receive range of the transmitting node switch to RTC status if their own HDTS is less than or equal to the HDTS of the sender (e.g., node 6, which has an HDTS of three, switches to RTC status; however, node 9, which has an HDTS of 5, does not). When a node switches to RTC mode, it starts to relay the data packets if it has data packets for the desired multicast group. If it does not have the desired data packets, it propagates the RTC request by broadcasting a RTC packet to its one-hop neighbours. This procedure continues until a node with the desired data packets is found The RTC mechanism is the last resort to fix a broken tree branch because 1) it takes more time to recognize such a significant collapse in the multicast tree and activate the RTC mechanism and 2) it requires more resources in terms of bandwidth and energy to fix (or in some cases, recreate) such tree breakages. Nevertheless, the other mechanisms are capable of creating, maintaining, and repairing the multicast tree without the use of the CRB mechanism in the majority of the situations. Hence, RTC is an infrequently utilized mechanism when compared to the other mechanisms, yet without it, MMC-TRACE is not a complete multicast protocol and cannot cope with network dynamics efficiently.

\subsection{SIMULATIONS AND RESULTS:}

We explored the QoS and energy dissipation characteristics of MMC-TRACE and MC-TRACE through extensive ns _ 2 simulations. The ns _ 2 MC-TRACE code provided by the $n s{ }_{-} 2$ Monarch wireless extensions. We have chosen MC-TRACE as the multicast protocol to compare with MC-TRACE because 1) MC-TRACE is a well-known multicast protocol for ad hoc networks, 2) There are many studies that compare OMC-TRACE against other group communication (multicast/broadcast) protocols, and therefore, it is possible to compare the performance of MMC-TRACE with many other protocols by benchmarking against MC-TRACE, and 3) we want to show that it is possible to reach the QoS level provided by a general purpose architecture (MC-TRACE) for voice communications with an energy conserving multicast architecture (MMC-TRACE). 
International Journal of Computer Science, Engineering and Information Technology (IJCSEIT), Vol.2, No.3, June 2012

The voice packet generation rate is set to $32.0 \mathrm{~ms}$, and the voice packet drop threshold (the maximum allowed lifetime of a voice packet) is set to $160.0 \mathrm{~ms}$. Furthermore, we used another packet drop threshold for the packets at the source (T drop - source 1/4 32:0 ms) for avoiding delay accumulation at the source.

The following graphs are resulted as the simulation results viz., as follows

1.Energy Dissipation

2.Delay

3.Packet Delivery Ratio

\begin{tabular}{|l|l|l|}
\hline Symbol & Parameter & Value \\
\hline$N / A$ & Number of nodes & $128 / 256$ \\
\hline$N / A$ & Network area & $1 \mathrm{~km} \times 1 \mathrm{~km}$ \\
\hline$N / A$ & Maximum node speed & $5.0 \mathrm{~m} / \mathrm{s}-25.0 \mathrm{~m} / \mathrm{s}$ \\
\hline$N / A$ & Multicast group size & 8 nodes $-256 \mathrm{nodes}$ \\
\hline$N / A$ & Transmit range & $250 \mathrm{~m}$ \\
\hline$N / A$ & Carrier sense range & $550 \mathrm{~m}$ \\
\hline$T_{d r o p}$ & Packet drop threshold & $160 \mathrm{~ms}$ \\
\hline$T_{\text {drop-source }}$ & Packet drop threshold at source & $32 \mathrm{~ms}$ \\
\hline$P_{T}$ & Transmit power & $600 \mathrm{~mW} / 2500 \mathrm{~mW}$ \\
\hline$P_{R}$ & Receive power & $300 \mathrm{~mW} / 900 \mathrm{~mW}$ \\
\hline$P_{I}$ & Idle power & $100 \mathrm{~mW} / 110 \mathrm{~mW}$ \\
\hline$P_{S}$ & Sleep power & $10 \mathrm{~mW} / 20 \mathrm{~mW}$ \\
\hline$C$ & Channel rate & $2 \mathrm{Mbps}$ \\
\hline$N / A$ & Data packet payload & $125 / 250 \mathrm{bytes}$ \\
\hline$T_{S F}$ & Superframe time & $32 \mathrm{~ms}$ \\
\hline$T_{R L Y}$ & Relay status expiration time & $5 T_{S F}$ \\
\hline$T_{A C K}$ & Downstream node expiration time & $2 T_{S F}$ \\
\hline$T_{C R B}$ & CRB time & $6 T_{S F}$ \\
\hline$T_{H D T S 1}$ & HDTS decrement time & $20 T_{S F}$ \\
\hline$T_{H D T S 2}$ & HDTS expiration time & $40 T_{S F}$ \\
\hline
\end{tabular}

Table 1: Simulation Parameters 


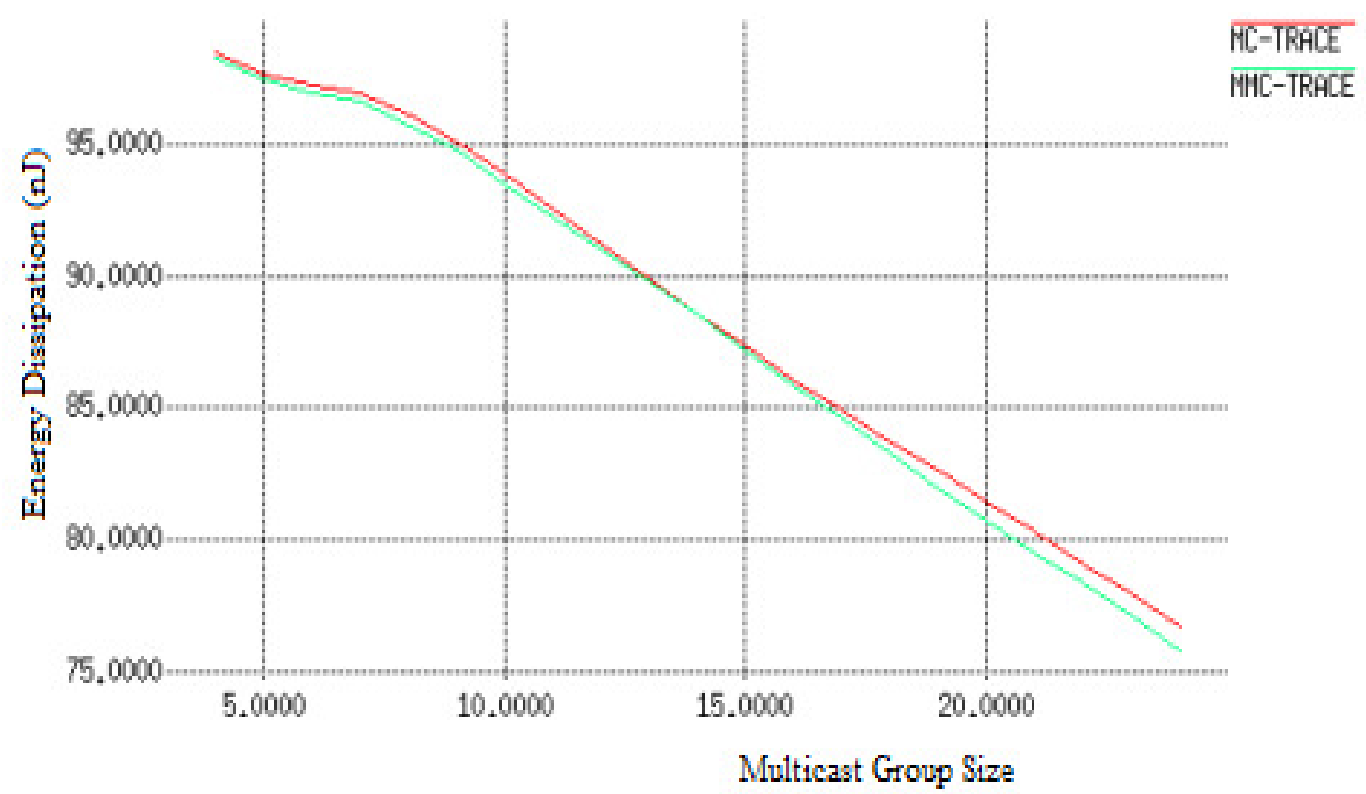

Figure 2: The Energy Saving Factor

As a first result we describe about the Energy Dissipation factor occurs in MANETs. While forming a Mobile Ad-Hoc Network there are various node which are spread over a wide area. So there will be exactly a source node and remaining as destinations. So here a packet from one node is send to another they form a path and travel that particular path. So in case of failure of one path the packets are travelled through another alternative path. While doing so the previous path remains active but their activation takes energy to be wasted. So here we implement the concept of switching the node to sleep mode by means of which the waste of energy is conserved .So in the following graph we represent a enough difference between the MMC-TRACE and MCTRACE by means of graphical representation. The $\mathrm{x}$-axis and $\mathrm{y}$-axis are taken as time and average energy respectively. For a small scale integration we take the time factor to be represented in milliseconds (ms) and the energy factor as Joules(J).Joules is a universal standard for representing energy and here the amount of energy conserved is considerably low. For a peculiar difference here MC-TRACE is represented as red plots and MMC-TRACE as green plots. Thus by means of graphical proofs MMC-TRACE is more energy efficient compared to MC-TRACE 


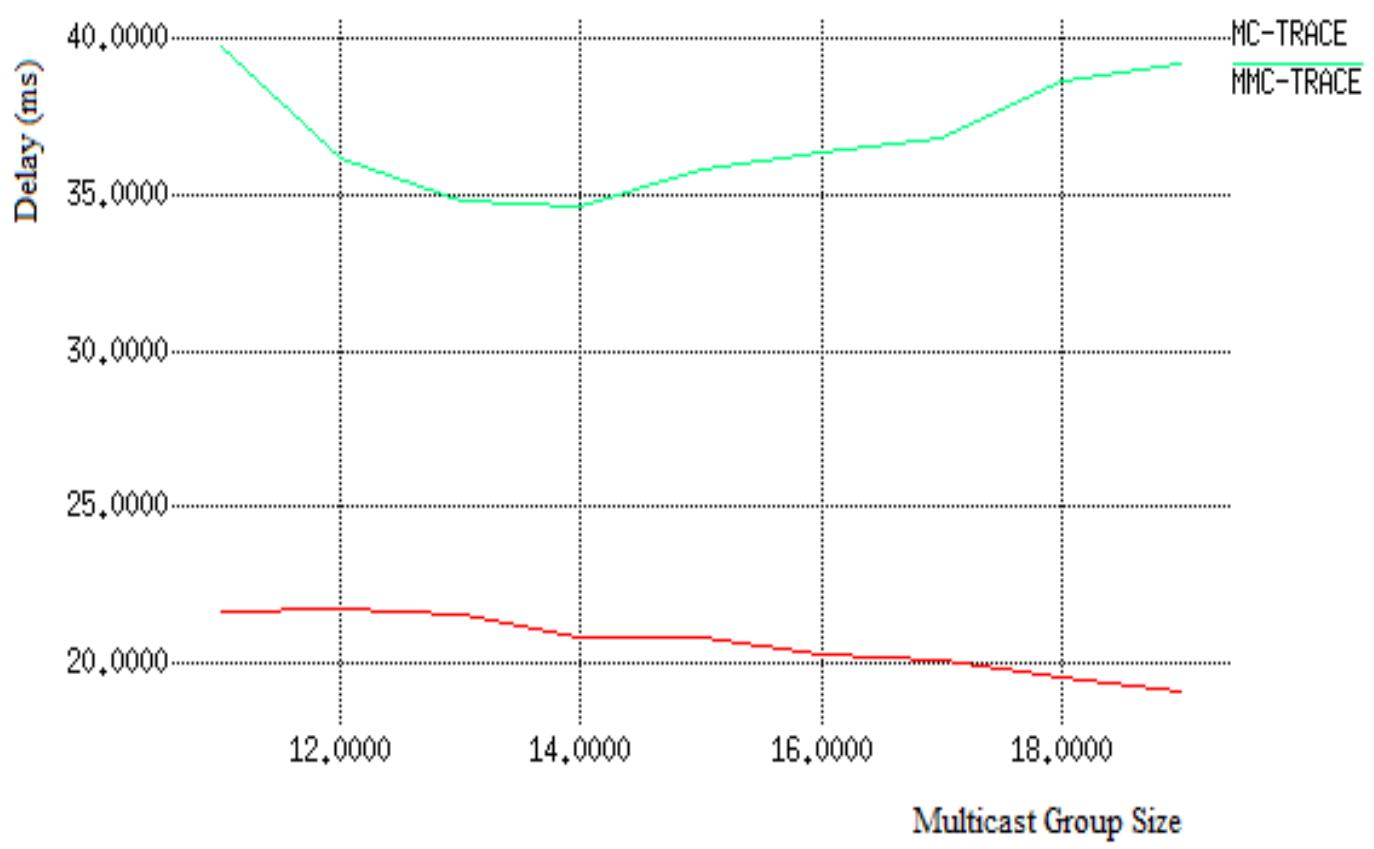

Figure 3:The Delay factor as per data transfer

In this section we describe about the delay factor taken place during the transmission of packets. As a general concept the data's from one node to another node are travelled by means of small packets as per the capacity of the route. While doing so the 1 st packet reaching the destination node must immediately send the acknowledgement to alarm the source to send the next packet. The time taken for the acknowledgement to reach the source is the factor called as delay. So delay could be reduce by reducing the interferences across the route node and making one node for one particular purpose. By means of which work maintained faster and this too involved in the energy conservation. Now the graphical representation denotes the comparison graphs between MMCTRACE and MC-TRACE. The x-axis and y-axis terms are taken as time and delay respectively. The differences are made by plotting with different colours .i.e. MMC-TRACE with green colour and MC-TRACE with red colour. Here the timing factor is represented in milliseconds(ms).Finally the difference graph states that MMC-TRACE have less delay compared to MC-TRACE.A common term that delay is taken a value with multiple of $10^{\wedge}-3$ because delay always remains low for a particular packet and they have its maximum state only during system hang up. 


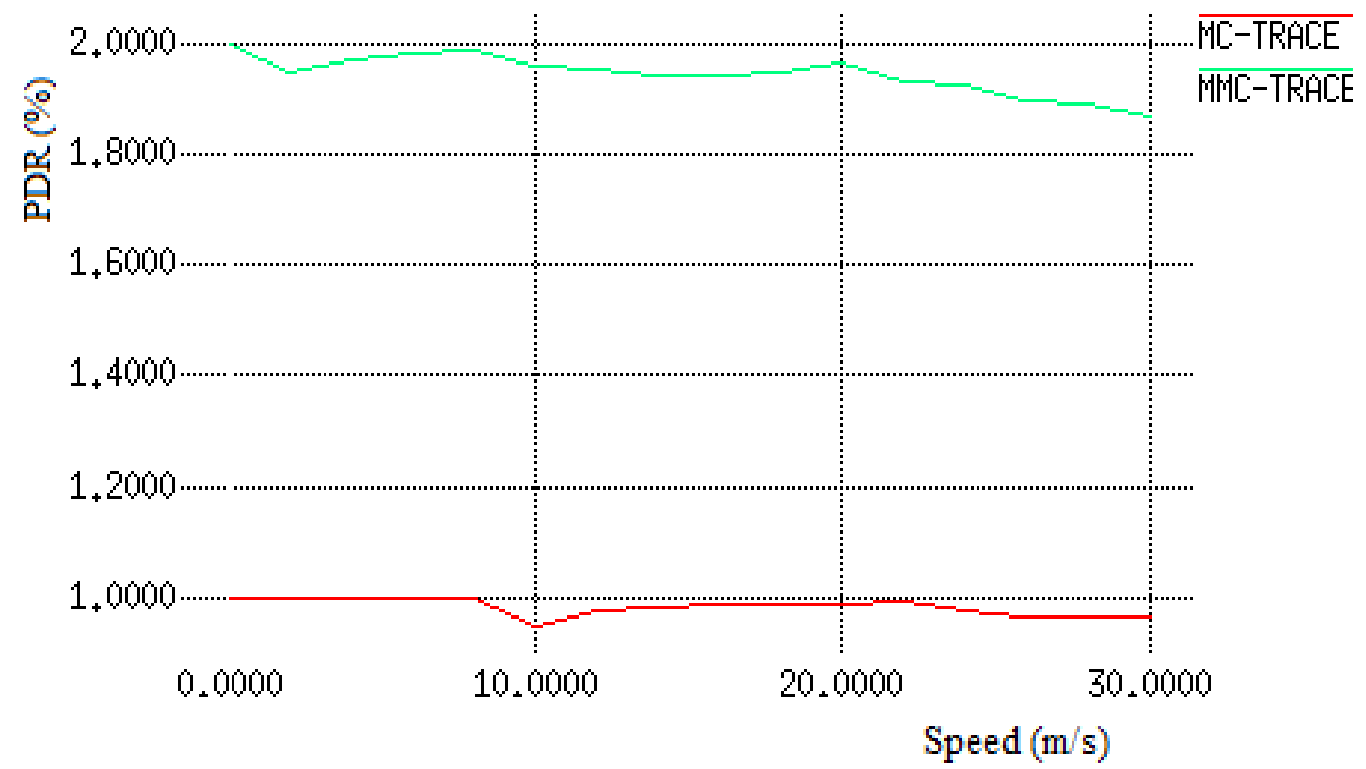

Figure 4: Packet Delivery Ratio Vs Speed

Third result of the simulation is considered to be Packet Delivery Ratio (pdr).And the following section briefly explains about pdr. As per the following representation graphs the difference graphs are drawn between MMC-TRACE and MC-TRACE and the colour representations as green and red respectively. As a general concept pdr means the overall ratio of the packets delivered to the particular destination. So by means of which a ratio is calculated. These values are plotted in a same graph for different values of MMC-TRACE and MC-TRACE with various representation. So the $\mathrm{x}$ and $\mathrm{y}$ terms are taken as time and pdr.Here the representations are made as milliseconds(ms) for time and no. of packets respect to pdr. The final statement states that the pdr level of MMC-TRACE is higher than MC-TRACE. So the time taken remains less and the energy factor is reduced. Do to this the Bandwidth efficiency is achieved. By means of all parameters a competitive Quality of Service is achieved.

\section{Conclusions}

We have compared the performance of MMC-TRACE with MC-TRACE in terms of packet delivery ratio, delay, and energy dissipation through ns-2 simulations. The packet delay of MMCTRACE is lesser than MC-TRACE for low data rate with low node density. But for high data rate with high node density for larger multicast group sizes, MMC-TRACE delay exceeds MCTRACE due to high congestion in the network. On the other hand, packet delivery ratio is higher than MC-TRACE at all data points. As the delay and packet delivery ratio are important metrics in real-time data QoS, from the above results it can be concluded that the competitive QoS performance has been provided with MMC-TRACE. It is a tree-based approach, yet it can preserve the tree branches in high mobility because it can detect broken tree branches rapidly, and with the support from the passively participating neighbouring nodes around the active branches, repair the broken links, mostly, locally. Thus, the well-known branch breakage vulnerability of tree-based multicast approaches in highly dynamic scenarios is alleviated by incorporating the passive condensed mesh concept into the tree-based multicasting. 
International Journal of Computer Science, Engineering and Information Technology (IJCSEIT), Vol.2, No.3, June 2012

\section{REFERENCES}

[1] Energy-Efficient Real-Time Multicast Routing in Mobile Ad Hoc Networks Bulent Tavli and Wendi B. Heinzelman, Senior Member, IEEE

[2] C.S.R. Murthy and B.S. Manoj, Ad Hoc Wireless Networks: Architectures and Protocols. Prentice Hall, 2004.

[3] . Janssen, D.D. Vleeschauwer, G.H. Petit, R. Windey, and J.M.Leroy, "Delay Bounds for Voice over IP Calls Transported over Satellite Access Links," Mobile Networks and Applications, vol. 7, pp. 7989, 2002.

[4] B.Tavli and W. Heinzelman, Mobile Ad Hoc Networks: Energy-Efficient Real-Time Group Communications. Springer, 2006.

[5] C.W. Wu and Y.C. Tay, “AMRIS: A Multicast Protocol for Ad Hoc Wireless Networks,” Proc. IEEE Military Comm. Conf., vol. 1, pp. 25-29, 1999.

[6] S.J. Lee, W. Su, and M. Gerla, "On-Demand Multicast Routing Protocol in Multihop Wireless Mobile Networks,” Mobile Networks and Applications, vol. 7, pp. 441-453, 2002.

[7] V. Kawadia and P.R. Kumar, "A Cautionary Perspective on Cross- Layer Design," IEEE Wireless Comm., vol. 12, no. 1, pp. 3-11, Feb.2005.

[8] J.G. Jetcheva and D.B. Johnson, "Adaptive Demand-Driven Multicast Routing in Multi-Hop Wireless Ad Hoc Networks,’Proc. ACM Int'l Symp. Mobile Ad Hoc Networking and Computing,pp. 33-44, 2001.

[9] S. Athanassopoulos, I. Caragiannis, C. Kaklamanis, and P.Kanellopoulos, "Experimental Comparison of Algorithms for Energy-Efficient Multicasting in Ad Hoc Networks," Proc. Int'l Conf. Ad-Hoc, Mobile, and Wireless Networks, pp. 183-196, 2004.

[10] W. Liang, "Approximate Minimum-Energy Multicasting in Wireless Ad Hoc Networks," IEEE Trans. Mobile Computing, vol. 5, no. 4, pp. 377-387, Apr. 2006.

[11] B. Tavli and W. Heinzelman, "TRACE: Time Reservation Using Adaptive Control for Energy Efficiency," IEEE J. Selected Areas Comm., vol. 21, no. 10, pp. 1506-1515, Dec. 2003.

[12] B. Tavli and W. Heinzelman, "MH-TRACE: Multi Hop Time Reservation Using Adaptive Control for Energy Efficiency,” IEEE J. Selected Areas Comm., vol. 22, no. 5, pp. 942-953, June 2004.

[13] B. Tavli and W. Heinzelman, "Energy and Spatial Reuse Efficient Network Wide Real-Time Data Broadcasting in Mobile Ad Hoc Networking," IEEE Trans. Mobile Computing, vol. 5, no. 10,pp. 1297-1312, Oct. 2006.

[14] H. Moustafa and H. Labiod, "A Performance Comparison of Multicast Routing Protocols in Ad Hoc Networks,” Proc. IEEE Personal, Indoor and Mobile Radio Conf., pp. 497-501, 2003.

[15] Network Simulator (NS), http://www.isi.edu/nsnam/ns, 2010.

[16] S.J. Lee, W. Su, J. Hsu, M. Gerla, and R. Bagrodia, "A Performance Comparison of Ad Hoc Wireless Multicast Protocols,” Proc. IEEE Conf. Computer Comm., pp. 565-574, 2000.

[17] W. Ye and J. Heidemann, "Medium Access Control in Wireless Sensor Networks," Technical Report ISI-TR-580, Information Sciences Inst., Univ. of Southern California, 2003.

[18] J.-P. Ebert, S. Aier, G. Kofahl, A. Becker, B. Burns, and A. Wolisz,"Measurement and Simulation of the Energy Consumption of an WLAN Interface," Telecomm. Network Group Technical Report TKN-02-010, Technical Univ. of Berlin, 2002.

[19] B. Tavli and W. Heinzelman, "MC-TRACE: Multicasting through Time Reservation Using Adaptive Control for Energy Efficiency,” Proc. IEEE Military Comm. Conf., pp. 2076-2081, 2005.

[20] M. Conti, G. Maselli, G. Turi, and S. Giordano, "Cross-Layering in Mobile Ad Hoc Network Design," Computer, vol. 37, no. 2, pp. 48- 51, Feb. 2004.

[21] M. Van Der Schaar and S. Shankar, "Cross-Layer Wireless Multimedia Transmission: Challenges, Principles and New Paradigms," IEEE Wireless Comm., vol. 12, no. 4, pp. 50-58, Aug. 2005. 


\section{Authors}

Sankar M S received the BE degree in electronics and communication engineering from the Anna University, Chennai, Tamil Nadu, India, in 2010, and awaiting for the ME degree in communication systems from Anna University, Chennai ,Tamil Nadu, India, in 2012 respectively.. His research interests include telecommunications, networking, signal processing, and image processing.

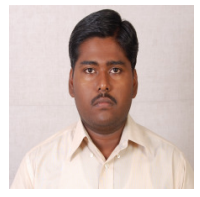

Suganthi.B received the B.E electronics and communication engineering from the Bharathidasan University, Tiruchirappalli, in 1999 and ME degree in communication systems from the Anna University, Chennai, Tamil Nadu, India, in 2007. Her research interest includes mobile computing.

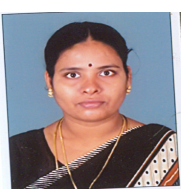

